\section{Competing interests None.}

Provenance and peer review Commissioned internally peer reviewed.

Thorax 2012;67:1-2

doi:10.1136/thoraxinl-2011-201340

\section{REFERENCES}

1. Crichton F, Ramsay CF, Pearson D, et al. Oral montelukast in acute asthma exacerbations: a randomised, double-blind, placebo-controlled trial. Thorax 2011;66:7-11
2. Moore RP, Berlowitz DJ, Denehy L, et al. A randomised trial of domiciliary, ambulatory oxygen in patients with COPD and dyspnoea but without resting hypoxaemia. Thorax 2011;66:32-7.

3. Churchyard GJ, Fielding K, Surita Roux S, et al. Twelve-monthly versus six-monthly radiological screening for active case-finding of tuberculosis: a randomised controlled trial. Thorax 2011;66:134-9.

4. Fischer BM, Mortensen J, Hansen $\mathrm{H}$, et al. Multimodality approach to mediastinal staging in nonsmall cell lung cancer. Faults and benefits of PET-CT: a randomised trial. Thorax 2011;66:294-300.
5. Perrin K, Wijesinghe M, Healy B, et al. Randomised controlled trial of high concentration versus titrated oxygen therapy in severe exacerbations of asthma Thorax 2011;66:937-41.

6. Christophe $\mathbf{M}$, Pison CM, Cano NJ, et al; The IRAD Investigators. Multimodal nutritional rehabilitation improves clinical outcomes of malnourished patients with chronic respiratory failure: a randomised controlled trial. Thorax 2011;66:953-60

7. Bush A, Pavord I. New Year resolutions. Thorax 2011;66:91-2

\title{
Is air pollution of the 20th century a cause of current asthma hospitalisations?
}

\section{Nino Künzli ${ }^{1,2}$}

Danish researchers followed $>57000$ older people over 10 years up to 2006. ${ }^{1}$ They made unique efforts to individually estimate exposure to traffic-related pollution for 35 years back in time, using modelled home-outdoor nitrogen dioxide $\left(\mathrm{NO}_{2}\right)$ concentrations. A $5.8 \mu \mathrm{g} / \mathrm{m}^{3}$ contrast in the 35-year average $\mathrm{NO}_{2}$ concentration was associated with a $10 \%$ higher risk for a first-ever hospitalisation due to asthma. In other words, pollution of the last century appears to contribute to current hospitalisations. But does this interpretation make sense? First, I will present arguments against this conclusion. Second, I will explain how to make sense of this large and well-conducted study.

To clarify the interpretation of the results, it is worth formulating two basic hypotheses relevant to research on air pollution and chronic diseases like asthma where underlying chronic pathologies (eg, hyper-reactive airways) are superimposed by acute expressions of the chronic disease (eg, asthma attacks). ${ }^{2}$ Under this model, two primary hypotheses emerge for exposure to air pollution:

- H1: Exposure supports the development of the underlying chronic pathology and, thus, increases the pool of people with chronic conditions (chronic effects) prone to exacerbations or 'events'

${ }^{1}$ Swiss Tropical and Public Health Institute, Basel, Switzerland; ${ }^{2}$ University of Basel, Basel, Switzerland

Correspondence to Dr Nino Künzli, Department of Epidemiology and Public Health, Swiss Tropical and Public Health Institute, Socinstrasse 57, PO Box, 4002 Basel, Switzerland; nino.kuenzli@unibas.ch
- H2: Exposure triggers an acute event (or a state of frailty that results in an event with a delay of a few days or weeks) among those with the disease (acute and subacute effects).

In $\mathrm{H} 2$, the underlying cause of the asthma cases may (H1) or may not be air pollution. While mechanisms related to the two hypotheses are not necessarily independent, there are possibly different causes and pathways involved in the two time domains of effects. For example, exposure to cold air may trigger an asthma attack, while living in a colder climate may not necessarily relate to higher incidence of asthma.

$\mathrm{H} 2$ has been well studied for a range of events (including hospital admissions) shown to be associated with the level of pollution in previous hours, days or few weeks. ${ }^{3} \mathrm{H} 1$ assumes instead that a chronic disease process is supported by long-term exposures to air pollution; therefore, classic $\mathrm{H} 2$ studies (eg, time series analyses and panel studies) with exposure terms derived for a few days or weeks do not address H1. The Danish study uses an estimate of (very) long-term exposure and, thus, the question arises whether it provides evidence for adult-onset asthma incidence $(\mathrm{H} 1) .{ }^{1}$ Investigating the chronic consequences of pollution requires indeed an estimate of long-term exposure; however, this is, by itself, not sufficient. $\mathrm{H} 1$ research also requires an appropriate 'chronic' health outcome. If H2 is true, I would argue that event-based outcomes (eg, asthma attacks, hospitalisation, death) are not the right choice to investigate $\mathrm{H} 1-$ no matter what data one may have at hand.
Related concepts have been discussed in interpreting mortality studies ${ }^{4}$ or chronic cardiovascular pathologies. ${ }^{2}$ Let me apply these issues to asthma.

Let us pretend that $\mathrm{H} 1$ cannot be possible; thus, all we need to investigate are the acute or subacute effects $(\mathrm{H} 2)$. While $\mathrm{H} 2$ can be well explored in classic 'acute effect studies', correlating yesterday's pollution with today's event frequencies, $\mathrm{H} 2$ could also be studied in cross-sectional surveys or in cohort studies even if the only available exposure term was some longterm mean concentration, such as in the study by Andersen et al. ${ }^{1}$ Under $\mathrm{H} 2$, 'period prevalence' of exacerbations or the frequency of hospitalisations must also be associated with the average 'long-term' concentration of pollution. This is not because the triggering of events would require cumulating exposures over months or years, but because a cleaner site has, on average, better air quality than a polluted site, both in the long term and in the short term. Accordingly, in clean sites air pollution related events are less frequent than in polluted places. To identify the relevant time windows of exposure one would need independent estimates of the various periods of exposure. The Danish study could-in theory-indeed investigate the independent effects of pollution during the week, month, season or year(s) prior to the hospital admission. In practice, this was not possible as the short-term exposure windows (days) prior to hospital admission were not available while the 1-, 15- and 35year estimates of 'long-term exposure' were so highly correlated that 'multiperiod' models could not be run. Conclusions made for the 35-year exposure also apply to the 15 years and the last 1-year mean (for which they observed the largest coefficients). As outlined above, the annual mean prior to the hospitalisation is expected to correlate with hospitalisations under $\mathrm{H} 2$ alone. In conclusion, the study confirms that air pollution affects asthma related hospital admissions but provides no 
evidence that current hospitalisation rates are affected by 'long-term' (35 years) exposures.

However, let me also present more useful albeit indirect interpretations. It is tempting to compare results of acute effect studies (based on classic designs such as time series or panel studies) with those (in my opinion, mislabelled) 'long-term studies' showing associations between events and longer term means of pollution. If $\mathrm{H} 2$ is true but $\mathrm{H} 1$ was null, the different studies should provide similar effect estimates per unit of pollution. However, if associations of pollution with 'events' are much larger in the 'long-term studies', it provides at least some indirect evidence in support of H1. Namely, if air pollution increases the pool of subjects with the chronic pathology (H1), more acute events are expected to be seen in polluted areas given that events (due to various causes) are part of the chronic disease career. Andersen et al provide the first 'long-term' study on asthma hospitalisations in adults that can be compared with 'acute effect' studies. The reported association between $\mathrm{NO}_{2}$ and 'first ever' hospitalisations is about 10-20 times larger in the Danish study than what classic acute effect studies observed. ${ }^{5}$ One indirect explanation for the discrepant results is that pollution not only triggered events but also increased the pool of asthmatics. This larger pool experiences more hospitalisations because of pollution but also due to many other causes. Effect estimates from cross-sectional and cohort studies capture this part of the burden, which is ultimately 'air pollution related' as well although there may be no causal link between air pollution occurring some 15-35 years ago and current hospital admission. Time series and panel studies do not assess those effects and are for that reason not informative in risk assessments where the total air pollution related burden is of interest. ${ }^{4}$

So far, $\mathrm{H} 1$ and $\mathrm{H} 2$ have been combined only in risk assessments of air pollution and childhood asthma, given the strong evidence of both $\mathrm{H} 1$ and $\mathrm{H} 2$ in children. ${ }^{6-8}$ Perez et al have shown that a very large burden of air pollution attributable exacerbations of childhood asthma relate not to the triggering per se (H1) but to air pollution increasing the pool of children with asthma. ${ }^{8}$ These children enter a 'chronic disease career' with its recurrent crises. Integrating the consequences of asthma incidence due to air pollution increases the burden attributable to air pollution twice for hospital admissions and some 10 times for bronchitis symptom episodes. ${ }^{8}$
As only few studies investigated the role of air pollution on adult-onset asthma, ${ }^{9}$ the indirect evidence that air pollution may affect the size of the pool of subjects with adult-onset asthma is currently of value. However, research is needed to investigate more directly the long-term role of pollution in the development of chronic asthmogenic pathologies (H1), and 'event based' studies are not the way to go as $\mathrm{H} 1$ and $\mathrm{H} 2$ are hardly disentangled. To understand the asthmogenic role of air pollution in adults and the relevant underlying pathways, we must have studies that measure features of the chronic pathologies and its development rather than the acute expression of the disease. This is a challenge for asthma-a label used for several phenotypes-characterised by a set of endotypes (or clusters of mechanisms) with probably different causes. ${ }^{10}$ It needs to be agreed on how to measure the development of asthma, how to identify the early stages of the pathology and, in more general terms, how to define and classify those phenotypes or endotypes. Occurrence of wheezing, bronchial reactivity or-as used in the Andersen study- hospitalisation is, per se, inadequate to describe the new onset of asthma. In the absence of agreed upon available early markers of the disease development, 'doctors' diagnosed asthma' may still serve its purpose despite the limitations related to differences in doctors' propensity to label patients as 'asthmatic'. 'Although 'doctors' diagnosed asthma' is based on symptoms as well, it is more specific than definitions based on wheezing or bronchial hyperreactivity alone, and more sensitive than the tip of the iceberg captured, if asthma onset was defined as a first-ever hospitalisation as done by Andersen et al. The characterisation of endotypes may shed more light on the possibly distinct phenotypes, its causes and the role of pollution. ${ }^{10}$ One disadvantage of using the incidence of doctors' diagnosed asthma is its definition as a dichotomous entity-you either have it or not. As in case of atherosclerosis, describing the phenotype on a continuous scale would be more appealing. ${ }^{2}$ Pekkanen et al have promoted this concept, which is further explored by Sunyer et al using a score, based on eight or five features, respectively, to describe the phenotype as a continuum. ${ }^{11}$ A high score had an extremely high specificity and positive predictive value for doctors' diagnosed asthma. Moving from a score of 0 to a score of $3+-$ possibly suggesting some proxy of asthma incidence-was associated with traffic-related air pollution in one study. ${ }^{13}$
However, there is still a long way to go to understand the endotypes of asthma and, thus, to unravel its specific causes.

Meanwhile, it is time to take policy action to improve air quality in our cities. Andersen et al very clearly confirm that traffic-related air pollution-even at rather low concentrations-poses serious respiratory health problems. ${ }^{1}$ Air pollution is preventable and evidence of health benefits of clean air policies is substantial. ${ }^{14}$

\section{Competing interests None.}

Provenance and peer review Commissioned; internally peer reviewed.

Published Online First 6 October 2011

Thorax 2012;67:2-3.

doi:10.1136/thoraxjnl-2011-200919

\section{REFERENCES}

1. Andersen ZJ, Bønnelykke K, Hvidberg M, et al Long-term exposure to air pollution and asthma hospitalizations in elderly adults: a cohort study. Thorax 2012;67:6-11.

2. Künzli N, Perez L, von Klot $S$, et al. Investigating air pollution and atherosclerosis in humans: concepts and outlook. Prog Cardiovasc Dis 2011;53:334-43.

3. Perez L, Rapp R, Künzli N. The Year of the Lung: outdoor air pollution and lung health. Swiss Med Wkly 2010;140:w13129.

4. Künzli N, Medina S, Kaiser R, et al. Assessment of deaths attributable to air pollution: should we use risk estimates based on time series or on cohort studies? Am J Epidemiol 2001;153:1050-5.

5. Peel JL, Tolbert PE, Klein M, et al. Ambient air pollution and respiratory emergency department visits. Epidemiology 2005;16:164-74.

6. Anderson R, Favarato G, Atkinson R. Long-term exposure to air pollution and the incidence of asthma: meta-analysis of cohort studies. Air Qual Atmos Health 2011; Published online first 7 April 2011. doi:10.1007/s11869-011-01445.

7. Künzli N, Perez L, Lurmann F, et al. An attributable risk model for exposures assumed to cause both chronic disease and its exacerbations. Epidemiology 2008;19:179-85.

8. Perez L, Künzli N, Avol E, et al. Global goods movement and the local burden of childhood asthma in southern California. Am J Public Health 2009; (99 Suppl 3):S622-8.

9. Jacquemin B, Schikowski T, Carsin A, et al. The role of air pollution in adult-onset asthma - a review of the current evidence. Sem Respir Crit Care Med. In press.

10. Lotvall J, Akdis CA, Bacharier LB, et al. Asthma endotypes: a new approach to classification of disease entities within the asthma syndrome. J Allergy Clin Immunol. 2011;127:355-60.

11. Pekkanen J, Sunyer J, Anto JM, et al. Operational definitions of asthma in studies on its aetiology. Eur Respir J 2005:26:28-35.

12. Sunyer J, Pekkanen J, Garcia-Esteban R, et al. Asthma score: predictive ability and risk factors. Allergy 2007;62:142-8.

13. Jacquemin B, Sunyer $\mathrm{J}$, Forsberg $\mathrm{B}$, et al Association between modelled traffic-related air pollution and asthma score in the ECRHS. Eur Respir $J$ 2009;34:834-42.

14. Künzli N, Perez L. Evidence based public health-the example of air pollution. Swiss Med Wkly 2009;139:242-50. 